\title{
Psychopathology and Assessment: Contributing Knowledge to Science and Practice
}

\author{
Randall T. Salekin
}

Published online: 11 February 2009

(C) Springer Science + Business Media, LLC 2009

This issue marks the beginning of my term as editor of the Journal of Psychopathology and Behavioral Assessment $(J P B A)$. I very much look forward to serving as the new Editor, and for the opportunity to build on the important work that has already been published under the founding editor Dr. Henry E. Adams, and Dr. Patricia B. Sutker, who served as editors for the past three decades (Sutker 2001). The articles that will appear in the next several issues will be manuscripts that were accepted under Dr. Sutker's editorship. The mission of the journal will be to continue the publication of high level scientific studies on psychopathology and assessment. I look forward to receiving manuscripts on both topics and believe that the two will continue to complement one another well in a single journal. Adams (1979) noted the importance of a multiassessment and paradigm approach stating that "this journal is planned to promote synthesis of assessment data from diverse theoretical perspectives and topic areas" (p. 1). I plan to continue with this agenda, and I interpret the use of the term "Behavioral" in the title of the journal to simply mean "Scientific." Thus, I hope to accept articles on both psychopathology and scientific assessment from multiple perspectives (biological, cognitive, behavioral, humanistic, etc.), and a variety of measurement sources (e.g., interview, self-report, other report, observation, imaging, etc). In addition, psychopathology that spans a wide variety of mental illnesses will be welcomed. I will also consider articles that emphasize well-being and positive aspects of psychology that have been found to prevent the development, or protect against the worsening of psychopathology. These articles will help to increase the field's knowledge of

\section{R. T. Salekin $(\bowtie)$}

Department of Psychology, The University of Alabama,

P. O. Box 870348, Tuscaloosa, AL 35487, USA

e-mail: rsalekin@bama.ua.edu psychopathology. They will aid in developing an understanding about how certain disorders come about (or do not come about), how they are maintained (or not maintained), and in cases where psychopathology develops, how the symptoms might be ameliorated.

\section{Will the Journal Emphasize Psychopathology or Assessment?}

$J P B A$ will publish articles on both psychopathology and assessment with no priority for either, the only emphasis will be on good science. Therefore, an article could emphasize psychopathology constructs and be accepted for publication. Alternately, an article could emphasize an assessment instrument(s) and also be accepted for publication. Finally, an article that reports on both psychopathology and instrumentation in a single manuscript can be accepted for publication, so long as it has sufficient focus. To elaborate on this point, articles often contain a wide range of psychometric instruments including self-reports, clinicians' ratings, structured or semi-structured interviews, and behavioral and physiological measures. Because of the broad scope of $J P B A$, authors have the opportunity to focus on the target constructs assessed by the instruments (e.g., depression, psychopathy, Conduct Disorder, Oppositional Defiant Disorder, anorexia, extraversion, generalized anxiety disorder, schizophrenia) and only briefly report on the psychometric properties of the test(s). Alternately, manuscripts can focus on instruments themselves, elucidating their strengths and weaknesses, and thus, the degree to which a particular measure is able to tap an underlying construct. Manuscripts that focus on instruments could be evaluated for their psychometric properties, such as scale homogeneity, inter-rater and test-retest reliability, item response functioning, and various aspects of validity, 
including ecological validity. The key to each submission is that the article be methodologically sound and have practical implications to be accepted for publication in the journal. Also, if research articles show that different measures result in considerably different effects, this too would be important to report, and for readers to know about.

Important knowledge can come from discovering differences across measures and sources. For instance, over two decades ago, Achenbach et al. (1987), when cumulating a large number of studies for his meta-analysis, showed substantial differences across child, adult, and teacher reports of psychopathology. This led researchers and clinicians to grapple with potential reasons for these differences (e.g., source bias, real differences across settings, etc), which ultimately led to a better understanding of the assessment of children and adolescents. Their findings, which elucidated discrepancies, were very practical, and helped researchers not only better understand the assessment of children, but resulted in new strategies for assessment and treatment. Thus, creative research ideas, sound designs, and appropriate statistical analyses and interpretations that are integrated into theoretical models can be critical in providing the foundations for delivering evidence-based practice in the future. Below, I discuss briefly some other potentially important considerations specific to psychopathology and assessment articles.

\section{Psychopathology Articles}

With respect to psychopathology, single- time point investigations can be helpful. At the most basic level of understanding, research often focuses on identifying whether variables are linked or correlated with a psychopathology (what Cronbach and Meehl (1955) referred to as the "nomological net"). The presence and magnitude of the relations among such variables can elaborate the nature of a problem and test or develop theories about its onset and the course of a disorder. This would be viewed as a basic, or preliminary, level of understanding. In psychopathology research, however, longitudinal research can give us an even richer understanding of a given disorder. Specifically, the notion of a risk factor represents a deeper level of understanding because the timeline is established, namely, that one event or experience (e.g., poor parenting practices), are correlated with a later characteristic or psychopathology. The early experience may not be a cause, but we know that the experience, for whatever reason, increased the likelihood of the outcome. Other distinctions can be made (see Kazdin 1999; Kraemer et al. 1997). If a risk factor is a stable characteristic that cannot be modified, or if modifying it has no effect on the outcome, it is sometimes referred to as a marker. Evaluating malleable risk factors might be helpful for psychopathologists and mental health professionals since these are the most likely to be relevant and useful for treatment. Some risk factors may play a causal role in the development of a psychopathology. Research studies that can demonstrate cause will help researchers and clinicians understand that the relation is not merely a temporal ordering of events, but rather a chain of direct influence that one event has on another. These types of studies will be particularly helpful in better understanding psychopathology (see Haynes 1992; Hill 1965; Schlesselman 1982).

Researchers and clinicians know well that there are many causes of psychopathology, so that identifying some event as a cause does not mean that event is the only cause. Research studies that investigate and report on different causal factors or multiple causal factors in a single article will be of great value. Research also often focuses on moderators, which are variables that influence direction, magnitude, and the nature of the relation (Baron and Kenny 1986; Kazdin 1999; McKinnon et al. 2007) and studies that examine the protective factors for particular disorders will also be considered for publication (see Rutter 1987; see also Salekin and Lochman 2008). Such studies shed light on questions such as this-Among individuals identified as at risk, many do not show the undesired outcome-why is this? The focus on mediators or mechanisms represents a deeper level of understanding beyond the relations noted previously because this means we know how the problem unfolds, through what processes, and the ways in which one variable leads to another (see Holmbeck 1997 and also Kazdin 1999; Salekin et al., in press). What mediates this relation, or through what mechanisms or processes does one characteristic (e.g., association with deviant peers) lead to the other (e.g., delinquent behavior)? Our understanding, of course, is optimal when we know all these answersspecifically, knowing the correlates, risk factors, moderators, causes and mechanisms or processes through which outcomes are produced. Studies that work toward this goal are very much encouraged and would greatly advance our understanding of psychopathology. This does not take away from the importance of continued cross sectional work, which will also be valued, but highlights the need in future years to also be working toward longitudinal psychopathology investigations that give researchers greater levels of knowledge about the risks, causes, and protective factors for a given disorder.

\section{Scope of Content: Will Articles Be Restricted to Only Certain Types of Disorders and Certain Age Groups?}

The journal will consider articles on a variety of disorders. As Editor, I hope to encourage an increase in the number of 
submissions on externalizing disorders which deserve greater representation. I would like to maintain the very strong emphasis on internalizing disorders, and at the same time, increase externalizing disorder research making both broad dimensions widely represented in the journal. Clearly, the better we understand all disorders across the DSM and other classification schemes, the better we will be able to treat a variety of conditions. In addition, studies that address the co-morbidity issues that cross internalizing and externalizing conditions will be welcomed.

With respect to participant age, traditionally $J P B A$ has leaned toward adult studies. However, I and the rest of the editorial staff are eager to consider research on psychopathology and test development and validation research across the life span. This would include young children, adolescents, young adults, and middle-aged and older adults. Recognizing that these are rough cuts, the journal is interested in publishing research across the life span, including developmental psychopathology research and testing innovations.

\section{Assessment Articles}

Scale development remains a growth industry within psychology. This can be observed through the pages of $J P B A$, as well as quick literature searches online for studies that entail the development and testing of psychological indices. As Clark and Watson (1995) put it, "assessment retains a central role within the field" (p. 309). Because testing remains a thriving activity and is chief to our understanding and treating of psychopathology, it is worthwhile discussing some important points about scale development and validation. It is my hope that this will help with designing studies, crafting articles, and ultimately maintaining and further enhancing the quality of the testing enterprise (see Clark and Watson 1995).

Although many researchers know that assessment instruments are supposed to be reliable and valid, the process of getting there is not always straightforward. Good words of advice for test construction and validity come from classic works by Cronbach and Meehl (1955), Loevinger (1957), and more recently, Clark and Watson (1995). With respect to Cronbach and Meehl (1955), they argued that investigating the construct validity of a measure entails, at least, the following steps: (a) articulating a set of theoretical concepts and their interrelations, (b) developing ways to index the hypothetical concepts proposed by a theory, and c) empirically testing the hypothesized relations among constructs and their observable manifestations. This means that without a specified theory (the nomological net), there is no construct validity. Such articulation of theory in the development and validation of tests for this journal would be, of course, welcomed. Moreover, a series of studies are needed to determine the construct validity of any measure, and these studies will need to entail more than a single set of observations. Rather than an isolated set of observations, studies must be conducted over time in order to examine the factor structure, links to other measures, differentiation between selected groups, and hypothesized changes over time or in response to a manipulation. These construct validity studies are critical in that the show us the way toward the most precise instruments.

Loevinger's (1957) monograph continues to be one of the most complete delineations of theoretically based psychological test construction. Watson and Clark elaborated on Loevinger's monograph suggesting the there are a number of important steps in test development and test validation. First, authors should consider whether a test is needed before developing a new test. If it is not needed, then researchers may want to devote time to some other knowledge gap in the literature. There are many reasons for new tests, such as the old ones do not work in the capacity for which they were designed, or they do not work as well as they should, or a test is needed for a different population, and so on. Loevinger (1957) and Clark and Watson (1995) provide excellent detail about item pool creation, distillation, and the subsequent validation process, and authors may benefit from refreshing their knowledge on these classic, and important, works prior to conducting their research and submitting articles.

\section{Interdisciplinary Research: General Models of Personality, Developmental Psychopathlogy, Biology and More...}

Research that is interdisciplinary can help with our understanding of the best practices in clinical psychology and particularly as they pertain to psychopathology and assessment. Cross-fertilization among disciplines will be encouraged for the betterment of psychopathology and assessment research. One recent example of cross fertilization is the research on personality and its potential relation to psychopathology (Widiger and Trull 2007). For instance, researchers have begun to suggest that psychopathology might be understood from the perspective of personality (see Caspi and Shiner 2006; Salekin and Averett 2008; Tackett 2006). Other research has begun to suggest that primary deficits (emotion regulation) may account for a host of disorders (Allen et al. 2008). Aside from these major lines of research, we also have DSM-V workgroups, grappling with taxonomic and dimensional models, as well as the possibility of reducing the number of categories for mental illness. The potential changes to the new manual could be vast and research that helps in DSM V decisions as 
well as tests new boundaries for mental illnesses will be of value. Research on the general models of personality, emotional deficits, and new DSM-V demarcations for disorders, illustrate just a few of the ways that multidisciplinary research could be helpful in gaining further resolution on psychopathology and its assessment. Articles that are interdisciplinary will be very much encouraged. There are many cross-fertilizations that are likely to be important, but given their recent prominence and their potential interest to authors, I mention two areas of study: behavior genetics and neuroimaging.

Behavior Genetics and the Environment (BG $x E$ ): Do we Really Need Traditional Psychological Assessment? Genes, and the extent to which they impact our lives, is a critically important issue. From the work of Darwin, Galton, and Mendel, and more recently Morgan, much has been learned. For instance, Morgan, with his famous Fly Room at Columbia University, was able to demonstrate that genes are carried on chromosomes, and are the mechanical basis of heredity. This finding formed the basis of the modern science of genetics. He was awarded the Nobel Prize in Physiology or Medicine in 1933, and was the first person awarded the Prize in genetics. Sir Alec John Jeffreys was also incredibly influential, with his studies leading to our understanding of DNA. Behavior genetics has greatly advanced our understanding of psychopathology. Much of this can be daunting to the psychopathology researcher who has typically asked questions of patients about how they think and feel. Despite huge advances in the area of behavior genetics, much work will be needed over the next few decades to advance our knowledge, and psychology certainly has an important role in what it can deliver with respect to both psychopathology and its assessment. Specifically, psychology can help out by developing precise measures that get us closer to the underlying construct being measured so that we are more certain about what is actually inherited from family member to family member. In addition, better measurement of environmental factors will be key if we are to understand what parts of the environment allow for the turning on and off of gene influences. Multidisciplinary research on psychopathology and its assessment and relation to behavior genetics will be encouraged.

FMRI Research and Task Development and Further Psychological Measurement. With the advent of the fMRI and "looking into the brain," we have gained great knowledge. However, our knowledge in this area can be furthered through the use of psychological assessment and pathology studies. Because neuroimaging research is so heavily dependent on specified tasks, a better understanding of what is being measured is very much needed. Thus, the psychological assessment enterprise could, in part, serve to develop tasks that are sophisticated and relevant to the question being asked. In addition, understanding what people are thinking and feeling in great detail, even as they are completing neuroimaging tasks, could be very helpful, and is a future avenue for researchers. Interconnecting psychological assessment with cognitive and biological paradigms will help to connect the disciplines and make many more real-life contributions with respect to neural activity and its psychological meaning within a given person (disorder), and within a certain context.

Other Important Innovations. Behavior genetics and neuroimaging are just a few areas that the field will need to intersect with, but other specific psychological assessment innovations and techniques could also advance the field of knowledge, such as daily diaries (emotion) and so forth. Research has shown that the daily monitoring of emotions can be an important source of information, and thus, much more research is needed on this topic. Longitudinal studies are incredibly helpful, but most researchers note that six-month gaps from one assessment point to the next could result in investigators missing important developmental information. Studies that look at day-to-day functioning across shorter time frames could also be critically important and augment other longitudinal work. The field needs both types of studies and fresh thoughts regarding how to answer many of the questions will be encouraged.

The willingness for cross-fertilization of research will mean that we, as psychologists and other mental health professionals, will need to continue to break out of the mold of being settled into our comfort zones, speaking only to those who speak our own language. In the past, we defined areas of common interest via society designations, divisions, conventions and so on. The fast pace of our field and the many discoveries to be made about psychopathology and assessment suggest the need for multidisciplinary research. Such research can mobilize action toward common causes resulting in very meaningful findings.

\section{Review Process}

The scope of this journal is broad in that it covers both psychopathology and assessment. As such, it requires consideration of articles on a wide range of topics using many different types of samples and various designs, instruments, and methodologies. Our team is looking forward to reading and reviewing papers, and providing knowledgeable and scholarly reviews of each manuscript. The editorial board has wide ranging expertise. A large number of top scholars have agreed to serve as either associate editor or editorial board members. Should any 
article require additional expertise that is not reflected on the board, we will seek additional reviewers with such knowledge.

Whether or not an article is accepted into the journal, we will strive to give authors constructive feedback. Sternberg (2003) has noted in the past that reviews of articles in psychological journals were often inappropriate and "have more of the characteristics of personal attacks than of a constructive critique of the work they are reviewing" (p. 159). We will discourage this practice and strive for professional and constructive reviews.

Finally, the journal will have speedy review process to increase the professionalism with which submissions are handled and, more important, to get scientists' research into press quickly. I recognize that authors will not submit their best work to the journal if the review process is lengthy. The journal is committed to having an initial decision on an article provided to authors on average, in 2 months. I will also be working toward having a short time between acceptance and seeing your article within the pages of JPBA.

\section{Special Sections and Issues to Increase Knowledge}

$J P B A$ will occasionally present research in the form of special sections to advance knowledge in areas that are topical and could benefit from a concentrated set of empirical studies. The journal will seek top scientists to prepare articles to advance knowledge on current and important topics. Authors will be encouraged to clearly articulate the immediate relevance of their findings, and to discuss where additional work is needed.

\section{Concluding Comments}

The primary goal of the Journal of Psychopathology and Behavioral Assessment is to publish articles that advance the science of psychopathology and assessment. This will be important for many reasons such as assisting with the DSM-V revision and beyond, and improving our understanding of the criteria and boundaries of disorders. As a journal with such an important role in science, it must place special demands on authors of the manuscripts that are considered. Again, the editorial staff is eager to assist individual authors in communicating the substance of their papers, or with less favorable editorial decisions, in identifying alternative approaches to the topic that will increase the likelihood of subsequent publication. It is an honor to be invited to serve as Editor for the journal and to continue to advance the course originally charted out by Henry E. Adams and Patricia B. Sutker. I, with the assistance of my editorial staff, shall strive toward excellence in the journal and welcome comments from readers and authors that are directed toward that specific goal.

\section{References}

Achenbach, T. M., McConaughy, S. H., \& Howell, C. T. (1987). Child/adolescent behavioral and emotional problems: Implications of cross-informant correlations for situational specificity. Psychological Bulletin, 101, 213-232.

Adams, H. E. (1979). Editorial. Journal of Behavioral Assessment, 1, $1-2$.

Allen, L. B., McHugh, R. K., \& Barlow, D. H. (2008). Emotional disorders: A unified protocol. In D. H. Barlow (Ed.), Clinical handbook of psychological disorders (4th Ed.). New York: Guiford Press.

Baron, R. M., \& Kenny, D. A. (1986). The moderator-mediator variable distinction in social psychological research: conceptual, strategic, and statistical considerations. Journal of Personality and Social Psychology, 51, 1173-1182.

Caspi, A., \& Shiner, R. L. (2006). Personality development. In W. Damon, R. Lerner, \& N. Eisenberg (Eds.), Handbook of child psychology: Social, emotional and personality development (vol. 3, pp. 300-365, 6th ed.). Hoboken, NJ: Wiley.

Clark, L. A., \& Watson, D. B. (1995). Constructing validity: Basic issues in objective scale development. Psychological Assessment, 7, 309-319.

Cronbach, L. J., \& Meehl, P. E. (1955). Construct validity in psychological test. Psychological Bulletin, 52, 281-302.

Haynes, S. N. (1992). Models of causality in psychopathology:Toward dynamic, synthetic, and nonlinear models of behavior disorders. Needham Heights, MA: Allyn \& Bacon.

Hill, A. B. (1965). The environment and disease: Association or causation? Proceedings of the Royal Society of Medicine, 58, 295-300.

Holmbeck, G. N. (1997). Toward terminological, conceptual, and statistical clarity in the study of mediators and moderators: Examples from the child clinical and pediatric psychology literatures. Journal of Consulting and Clinical Psychology, 65, 599-610.

Kazdin, A. E. (1999). Current (lack of) status of theory in child and adolescent psychotherapy research. Journal of Clinical Child Psychology, 28, 533-543.

Kraemer, H. C., Kazdin, A. E., Offord, D. R., Kessler, R. C., Jensen, P. S., \& Kupfer, D. J. (1997). Coming to terms with the terms with the terms of risk. Archives of General Psychiatry, 54, 337-343.

Loevinger, J. (1957). Objective tests as instruments of psychological theory. Psychological Reports, 3, 635-694.

MacKinnon, D. P., Fairchild, A. J., \& Fritz, M. S. (2007). Mediation analysis. Annual Review of Psychology, 58, 593-614.

Rutter, M. (1987). Psychosocial resilience and protective mechanisms. American Journal of Orthopsychiatry, 57, 316-331.

Salekin, R. T., \& Averett, C. (2008). Personality in childhood and adolescence. In M. Hersen, \& A. M. Gross (Eds.), Handbook of clinical psychology children and adolescents (vol. 2, (pp. 351385)). Hoboken, NJ: John Wiley \& Sons.

Salekin, R. T., \& Lochman, J. E. (2008). Child and adolescent psychopathy: The search for protective factors. Criminal Justice and Behavior, 32, 159-172.

Salekin, R. T., Rosenbaum, J., Lee, Z., \& Lester, W. (in press). Child and adolescent psychopathy: Like a painting by Monet. Youth Violence and Juvenile Justice.

Schesselman, J. J. (1982). Case-control studies: Design, conduct and analysis. New York: Oxford. 
Sternberg, R. J. (2003). There is no place for hostile reviews. International Journal of Clinical and Health Psychology, 3, $159-161$.

Sutker, P. B. (2001). A tribute to Henry Earl Adams (June 16, 1931October 16, 2000). Journal of Psychopathology and Behavioral Assessment, 23, 1-2.
Tackett, J. L. (2006). Evaluating models of the personality-psychopathology relationship in children and adolescents. Clinical Psychology Review, 26, 548-599.

Widiger, T. A., \& Trull, T. J. (2007). Plate tectonics in the classification of personality disorders. American Psychologist, $62,71-83$. 